Accretion Phenomena and Related Outflows, IAU Colloquium 169

ASP Conference Series, Vol. 121, 1997

D.T. Wickramasinghe, L. Ferrario, and G.V. Bicknell, eds.

\title{
Modelling the Polarization from Accretion Disks
}

\author{
O. J. Dittmann \\ Institut für Theoretische Astrophysik der Universität Heidelberg, \\ Tiergartenstr. 15 D-69121 Heidelberg, Germany
}

\begin{abstract}
The 3D transfer equation for polarized radiation is solved for several different model configurations. In the case of Rayleigh scattering the phasematrix is decomposed into a sum of matrix products to allow the use of operator splitting iteration schemes. For 30 slabs with constant extinction matrix and constant emission the emerging polarized fluxe are calculated. We find that the optical depth and the scattering parameter can be fixed from observations even if only the fluxes are known. Specifying the extinction matrix our models can be applied to the inner regions of active galactic nuclei.
\end{abstract}

\section{Introduction}

A great variety of objects of astrophysical interest exhibit polarization of their radiation. Of particular interest is the class of HPQs (high polarized quasars), that shows degrees of polarization up to $3 \%$ (Stockman et al., 1984). The cause for the polarization of these objects is probably scattering of the radiation in a nonspherical atmosphere. Seyfert galaxies have a thick surrounding dust torus. The distinction between type 1 and type 2 Seyferts is then due to a different viewing angle. In both these examples a deviation from spherical symmetry plays an important role.

The fundamental formulation of the transport equations dates back to Chandrasekhar (1950). Multidimensional numerical techniques for the solution of the transfer equation separate roughly into three categories. Approximate methods like the Sobolev method (Rybicky and Hummer, 1983; Jeffery, 1989), Monte-Carlo-Methods (Höflich, 1991) and accurate solutions (Stenholm and Stenflo, 1978; Auer, Heasley and House, 1977). None of these techniques can fully and accurately solve the polarized radiative transfer problem in three dimensions. The need for an efficient treatment of the multidimensional equation of polarized radiative transfer is then apparent.

\section{3D Transport of Polarized Radiation}

The polarized radiation field is described by the Stokes parameters $I, Q, U, V$. The three dimensional ERT has the form:

$$
\mathbf{n} \nabla \underline{I}=-(\underline{\kappa}+\underline{\sigma}) \underline{I}+\underline{\sigma} \int_{\Omega} \underline{P}\left(\Omega, \Omega^{\prime}\right) \underline{I}\left(\Omega^{\prime}\right) \frac{d \Omega^{\prime}}{4 \pi}+\underline{\kappa} B \underline{1}
$$


where $\underline{\kappa}$ is the absorption matrix, $\underline{\sigma}=\sigma_{I} \underline{E}$ is the scattering matrix and $\underline{P}\left(\Omega, \Omega^{\prime}\right)$ the phasematrix of the scattering process in question. $E$ denotes the four dimensional unity matrix and $\underline{1}=\left[\begin{array}{cccc}1 & 0 & 0 & 0\end{array}\right]^{T}$ is the unpolarized unit vector. Here we will only discuss the Rayleigh phasematrix, but almost all results can be generalized to arbitrary scattering processes.

\subsection{The Matrix Exponential Method}

For a given source function $\underline{S}$ the ERT along a single line of sight takes the form $\frac{\mathrm{d} I}{\mathrm{~d} \tau}=-\underline{\eta} \underline{I}+\underline{S}$. Since we consider polarization effects, $\underline{\eta}$ is a $4 \times 4$ matrix. We now just have to subtitute in the formal solution (Eq.2)

$$
\underline{I}(0)=\exp (-\underline{\eta} \tau) \underline{I}(\tau)-\exp (-\underline{\eta} \tau) \int_{0}^{\tau} \exp \left(\underline{\eta} \tau^{\prime}\right) \underline{S}\left(\tau^{\prime}\right) d \tau^{\prime}
$$

the scalar exponential function by the matrix exponential, where we define this quantity (cf. Kincaid and Cheney, 1991) by the usual series expansion. It can be written as a simple sum of powers of a single depth-independent matrix.

$$
\exp (\underline{\eta} \tau)=\sum_{i=0}^{3} c_{i}(\tau)\left(\underline{\eta}-\eta_{I} \underline{E}\right)^{i}
$$

with scalar depth-dependent coefficients $c_{i}(\tau)$.

If we assume that the source function varies liearly with optical depth (Unno solution, Unno 1956), we find a very simple formulation using the matrix exponential function (Dittman, 1996b). This onedimensional solution can be used as building block for the more complex.

\subsection{Numerical Solutions of the ERT}

A direct solution of the ERT would lead to a linear system of about $10^{8}$ unknowns. Even in the gray case the requirements on memory would be extreme. Therefore it is easier to first derive the solution of the ERT for a known source function and then iterate over the mean intensity until convergence is achieved. In this way angular and positional space decouple. This is only possible with a decomposition of the phasematrix. Therefore we split $\underline{P}$ into a sum of three matrix products, where each factor depends only upon $\Omega$ or $\Omega^{\prime}$. $\underline{P}\left(\Omega, \Omega^{\prime}\right)=\sum_{i=0}^{2} \underline{A}_{i}(\Omega) \underline{A}_{i}^{T}\left(\Omega^{\prime}\right)$, where $\underline{A}_{i}^{T}$ is the transpose of $\underline{A}_{i}$ (Dittmann, 1995).

As a discretization scheme we use the short characteristics technique by Olson et al (1986) and as an iteration technique we choose an operator pertubation approach with the diagonal matrix as the approximate operator. Because of the the splitting of the phasematrix into three components, we also have to iterate for three mean intensities $\underline{J}_{i}=\int \underline{A_{i}}\left(\Omega^{\prime}\right) \underline{I}\left(\Omega^{\prime}\right) d \Omega^{\prime}$.

\section{Results}

The aim of this work is to investigate systematically the dependence of the solution of the ERT on the form of the region under consideration and the magnitude of scattering. Therefore we restrict ourselves to the computation of boxes 
with the following parameters: $\varepsilon \in\{0.01,0.1,0.5,1\}, \tau_{z} \in\{0.1,1,10\}, b_{1}=\frac{\tau_{x}}{\tau_{z}} \in$ $\{0.01,0.1,1\}$ and $b_{2}=\frac{\tau_{y}}{\tau_{z}} \in\{0.01,0.1,1\}$. The discretisation has 41 gridpoints in each spatial and 16 in each angular direction. Furthermore we neglect magnetooptical effects and take the absorption matrix as constant. We choose $\eta_{I}=1$ and $\eta_{Q}=\eta_{U}=\eta_{V}=0.3$. The emission is also constant and unpolarized and only Rayleigh scattering is considered.

\subsection{Optical Depth and Scattering Parameter}

Fluxes
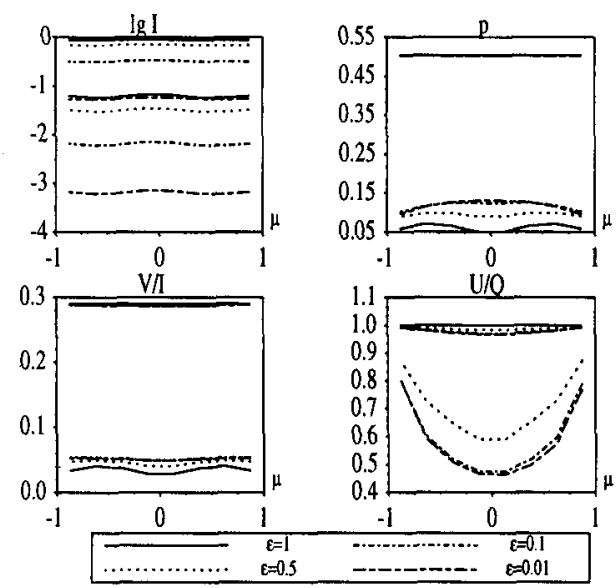

Fluxes
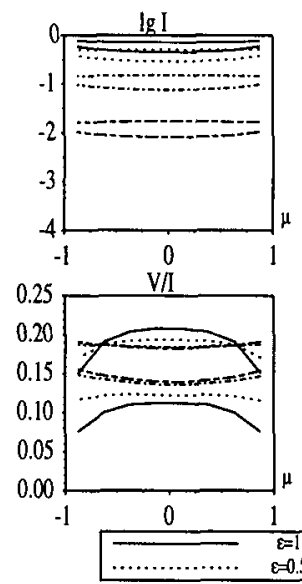
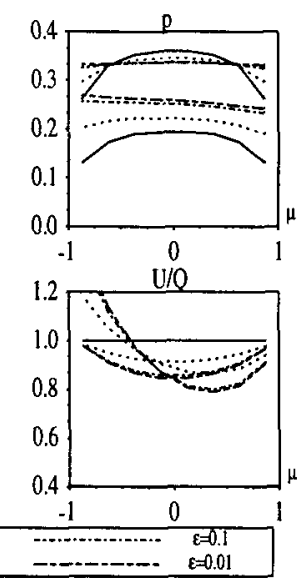

Figure 1. Integrated Stokes vector, degree of polarization, angle of polarization and ellipticity for different geometries (s. text) as a function of the viewing angle.

We calculated the fluxes, because AGN often cannot be spatially resolved. The upper set of lines in the left picture of fig. 1 shows the optically thin cube the lower set the optically thick cube. Since the emisson scales with the scattering parameter, the absolute values of the Stokes parameters drop accordingly. The degree of polarization is almost constant at $\bar{p} \approx 0.50$ for the thin cube and significantly lower for the thick cube. The latter shows a rise of $p$ as scattering becomes more important. The quantity most sensitive to the viewing angle is the tangent of the angle of polarization. Even in the optically thin case it might be used to tell different models apart.

\subsection{Disks and Jets}

The right part of fig 1 compares a disklike with a jetlike structure. The lower set of different scattering parameters belongs to the jet, the upper to the disk. Again we find that the optically thinner jet has a markedly higher degree of polarisation. The most striking difference is the rotation of the polarisation angle for viewing from the bottom. In principle this effect can be used to determine the orientation of an asymmetric object.

One can also analyse the whole range of different configurations we calculated. The geometry is parametrized with $\lg b_{1}$ and $\lg b_{2}$, the greatest optical 
thickness is fixed at $\tau_{z}=10$. We find that the dependence of the Stokes parameters on the scattering parameter can for all configurations be represented by: $\underline{I}\left(\Omega, \varepsilon, b_{1}, b_{2}, \tau_{z}\right) \approx \underline{C}\left(\Omega, b_{1}, b_{2}, \tau_{z}\right) \varepsilon^{0.97 \pm 0.03}$ (Dittmann, 1996a).

\section{Discussion}

We have shown that the three dimensional polarized equation of radiative transfer can numerically be solved. It should be emphasized that the assumptions used in our models are made only to investigate the parameters in a systematic fashion. The algorithms can very well treat magneto-optical effects and spatial dependence of the extinction matrix. Any illumination from the outside may be specified and the inclusion of different scattering processes can also be done. Finally the treatment of resonance lines is incorporated in the method, while the calculation of a full spectrum with many lines is still beyond the current computation power.

However even with these simplifications we find some interesting results. The main contribution to the polarization arises in optically thin regionsand even in configuration where only one side is optically thin, this one dominates the polarization properties. Scattering on the other hand strengthens the polarization from optically thick regions but the dominant parameter is the optical depth along a line of sight. Scattering contributes only as a secondary but measurable effect. The angle of polarization in disk-like structures is rotated counterclockwise, whereas it normally rotates clockwise. For not to small scattering parameters the fluxes depend almost linearly on $\varepsilon$.

With these results new astrophysical problems become treatable. In particular the high polarized AGN bear further investigation. Because of the cylindrical symmetry of the accretion disks at least a two dimensional model is needed. These models can be used to shed some new light on the unified model.

\section{References}

Auer L. H., Heasley J. N., House L. L. 1977, Solar Physics, 55, 47

Chandrasekhar S. 1950, Radiative Transfer, Oxford University Press

Dittmann O. J. 1995, Phd-thesis, www.ita.uni-heidelberg.de, Heidelberg

Dittmann O. J. 1996a accepted for JQSRT

Dittmann O. J. 1996baccepted for JQSRT

Höflich P. 1991, A\&A, 246,481

Jeffery D. J. 1989, ApJS, 71, 951

Kincaid D., Cheney W. 1991, Numerical Analysis, Brooks/ Cole Publ. Comp., Belmont

Olson G. L., Auer L. H., Buchler J. R. 1986, JQSRT, 35,431

Rybicki G. B., Hummer D. G. 1983, ApJ, 274, 380

Stenholm L. G., Stenflo J. O. 1978, A\&A, 67, 33

Stockman H. S., Moore R. L., Angel J. R. P. 1984, ApJ, 279,485

Unno W., 1956 PASJ, 8,108 